\title{
TESTES NEUROPSICOLÓGICOS DE APLICAÇÃO SIMPLES PARA O DIAGNÓSTICO DE DEMÊNCIA
}

\author{
RICARDO NITRINI*, BEATRIZ HELENA LEFÈVRE*, SANDRA CRISTINA MATHIAS*, \\ PAULO CARAMELLI*, PAULO EDUARDO M. CARRILHO*, NAIM SAUAIA **, EDUARDO MASSAD**, \\ CLÓVIS TAKIGUTI ***, IVALDO OLIMPIO DA SILVA**, CLÁUDIA SELLITO PORTO*, \\ MARIA CRISTINA MAGILA*, MILBERTO SCAFF*
}

\begin{abstract}
RESUMO - Os desempenhos em testes neuropsicológicos de trinta pacientes, com diagnóstico de demência leve ou moderada baseado nos critérios do DSM-III-R, com escore no Mini-exame do Estado Mental inferior a 24 pontos e escolaridade mínima de 4 anos, foram comparados aos de trinta voluntários normais equiparados quanto a idade, escolaridade e sexo. Foram calculadas as sensibilidades e especificidades dos testes na distinção entre doentes e controles normais Dentre os testes empregados, o que demonstrou maior acurácia nesta distinção, calculada através de curva ROC, foi o de informação-memória-concentração de Blessed, seguido em ordem decrescente de acurácia, pelos de construção (cópias de figuras simples), memória verbal tardia (após 5 minutos), reconhecimento de 10 figuras e fluência verbal (animais). Seis testes de aplicação e interpretação simples (fluência verbal, percepção visual, memória visual incidental, cálculo, desenho de um relógio e memória tardia após 5 minutos) permitiram definir função linear discriminante que revelou elevada capacidade de discriminação entre pacientes e controles, na amostra. Esta função deve ser submetida à comprovação em outra casuística. Em virtude da simplicidade e rapidez de aplicação, poderá ser associada ao MEM para identificar indivíduos com demência e indivíduos sadios em estudos epidemiológicos.
\end{abstract}

PALAVRAS-CHAVE: demência, avaliação, testes neuropsicológicos.

\section{Brief and easy-to-administer neuropsychological tests in the diagnosis of dementia}

SUMMARY - Thirty patients with dementia defined by DSM-III-R criteria (Alzheimer's disease (22), vascular dementia (3), Parkinson's disease, frontal lobe dementia, possible diffuse Lewy body dementia, normal pressure hydrocephalus and uncertain diagnosis), with scores below 24 points in the Mini-Mental Status Examination and more than 4 years of education were submitted to a neuropsychological evaluation. The scores in the neuropsychological tests were compared to those obtained by thirty normal volunteers paired for age, sex and education. Sensivity, specificity and accuracy of the tests in the distinction of demented and normal volunteers were determined. The accuracies were calculated using ROC curves. Blessed's information-memory-concentration test showed greatest accuracy, followed by copy of simple figures, delayed memory of 10 figures (after 5 minutes), recognition of 10 figures and verbal fluency test (animals). A linear discriminant function, composed by 6 tests: visual perception, incidental memory, delayed memory (after 5 minutes), drawing of a clock, verbal fluency (animals) and calculation tests, was able to discriminate all controls from patients and only one patient was wrongly classified as normal control. These tests were chosen because they can be applied in less than 10 minutes and are very easy to interpret. This discriminant function must be applied in another group of patients and controls in order to demonstrate its value. When associated to the MMSE it may be useful to discriminate patients with dementia from normal people in epidemiological studies.

KEY WORDS: dementia, evaluation, neuropsychological tests.

Faculdade de Medicina da Universidade de São Paulo: Departamentos de Neurologia*, Informática Médica** e Medicina Preventiva***. Aceite: 14-abril-1994.

Dr. Ricardo Nitrini - Rua Bartolomeu Feio 560 - 04580-001 São Paulo SP - Brasil. 
O diagnóstico de demência depende da avaliação do estado mental. Diversos testes, desde os de aplicação rápida até extensas baterias neuropsicológicas podem ser utilizados para essa avaliação. Todavia, não existe teste padrão-ouro para o diagnóstico. Testes de aplicação rápida e interpretação simples são de interesse no exame individual de pacientes, mas são ainda mais importantes para estudos epidemiológicos de prevalência de demência em populações. Entre esses, o Mini-exame do Estado Mental (MEM) ${ }^{9}$ tem sido o mais empregado. Não deve, entretanto, ser utilizado como único critério para o diagnóstico, principalmente pela especificidade relativamente baixa em estudos populacionais ${ }^{2}$; inversamente, sua sensibilidade é baixa na detecção de demência em indivíduos com escolaridade elevada ${ }^{26}$. A associação de outros testes ao MEM podem aumentar sua acurácia diagnóstica ${ }^{10,29}$. Com o objetivo de permitir avaliação rápida e relativamente precisa do estado mental, Storandt et al. ${ }^{21}$ propuseram a aplicação de 4 testes que, em 10 minutos, foram capazes de classificar corretamente $98 \%$ dentre 42 doentes com doença de Alzheimer e 42 controles; os testes empregados foram os de memória lógica e controle mental da Escala de Memória de Wechsler ${ }^{27}$, a forma A do teste de conexão de números ${ }^{4}$ e um teste de fluência verbal. Estes achados foram confirmados por estudo posterior ${ }^{25}$. De modo semelhante, Eslinger et al. $^{7}$ propuseram a combinação de 3 testes: retenção visual, associação de palavras e orientação temporal. Obtiveram sensibilidade e especificidade elevadas ( 87 e $91 \%$, respectivamente) quando avaliaram 53 pacientes com demência e 53 indivíduos do grupo controle. $O$ tempo de aplicação foi de 10 a 15 minutos.

Os objetivos deste estudo foram os de verificar quais testes de aplicação e interpretação simples apresentam maior acurácia na discriminação entre pacientes com demência e indivíduos normais e de avaliar a capacidade de um conjunto desses testes para esta discriminação, em nosso meio.

\section{CASUÍSTICA E METODOLOGIA}

Trinta pacientes com diagnóstico de demência leve ou moderada baseado nos critérios do DSM-III- $\mathbf{R}^{\mathbf{1}}$, com escore no MEM inferior a 24 pontos e escolaridade mínima de 4 anos foram selecionados dentre 100 pacientes atendidos no Ambulatório de Demência da Clínica Neurológica do Hospital das Clínicas da Faculdade de Medicina da Universidade de São Paulo ou na clínica privada do primeiro autor. Quanto ao diagnóstico, 22

Apêndice 1. Identificação de 10 figuras.

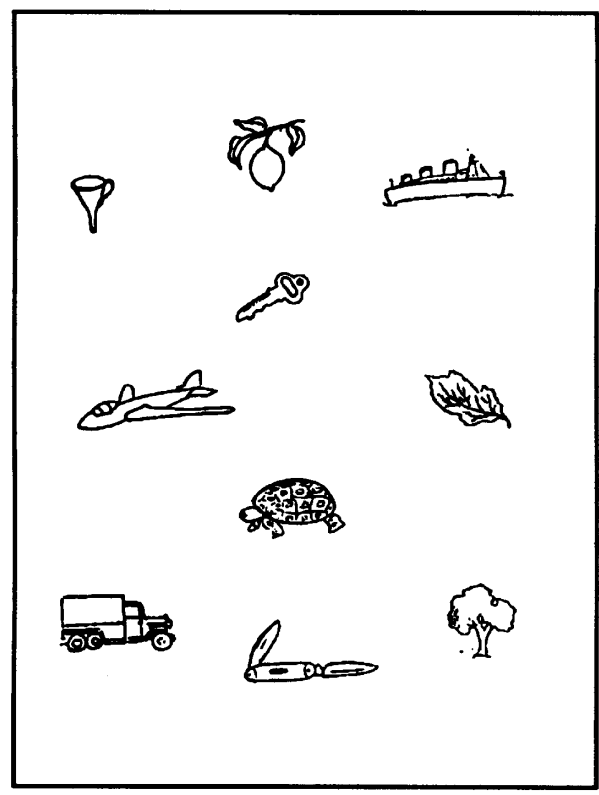
apresentavam doença de Alzheimer, 3 tinham demência vascular e entre os 5 cinco restantes havia 1 caso de cada um dos seguintes: demência do tipo frontal, doença de Parkinson, possível demência com corpúsculos de inclusão de Lewy disseminados, hidrocefalia a pressão normal e diagnóstico indeterminado. $O$ diagnóstico de demência de Alzheimer baseou-se nos critérios do National Institute of Neurological and Communicative Disorders and Stroke - Alzheimer's Disease and Related Disordes Association (NINCDSADRDA) ${ }^{13}$. O diagnóstico de demência vascular obedeceu aos critérios propostos pelo National Institute of Neurological Disorders and Stroke e pela Association Internationale pour la Recherche et l'Enseignement en Neurosciences (NINDS-AIREN) ${ }^{19}$. O diagnóstico de possível demência com corpúsculos de Lewy disseminados baseou-se em critérios sugeridos por Crystal et al. ${ }^{5}$ e McKeith et al. ${ }^{12}$.

A avaliação neuropsicologica, aplicada segundo a técnica empregada no Ambulatório de Demências ${ }^{18}$ foi constituída de: MEM, teste de Blessed ${ }^{3}$, repetição de dígitos (digit span) na ordem direta e na ordem inversa, teste de conexão de números (modificação do trail-making, $A)^{4}$, teste de fluência verbal (número de animais nomeados em 1 minuto), teste de percepção 
visual (identificação das 10 figuras constantes do Apêndice 1), testes de memória (memorização das 10 figuras identificadas, com avaliação da memória incidental, memória imediata, memória após latência de 5,15 e 30 minutos e reconhecimento e memória para fatos remotos), exames da linguagem, da capacidade de realizar cálculos numéricos (veja Apêndice 2), da habilidade construtiva, da capacidade de abstração, das gnosias (táctil, visual, digital e orientação direita-esquerda), das praxias, da capacidade de planejamento (teste de organizaçăo dinâmica de movimentos ${ }^{11}$, teste pare-siga ${ }^{28} \mathrm{e}$ desenho do relógio ${ }^{23}$ ). Os desempenhos dos pacientes nos testes neuropsicológicos foram comparados aos de indivíduos de grupo controle constituído de 30 indivíduos normais, pareados quanto a sexo, idade e escolaridade com os pacientes. Para participar do grupo controle, o indivíduo precisava estar em condições de cuidar de si próprio e de seus afazeres, ter escolaridade mínima de 4 anos, não apresentar sintomas e sinais clínicos de doença que estivesse afetando o SNC e não estar utilizando medicamentos tranquilizantes ou antidepressivos. Doenças crônicas como hipertensão arterial, diabetes melito e cardiopatia não foram critérios de exclusão, desde que compensadas.

Apêndice 2. Modo de aplicação dos testes utilizados na função discriminante.

1. Percepção Visual. Mostre a folha que contém as 10 figuras (figura 1A) e pergunte: "Que figuras são estas?".O escore é dado pelo número de figuras corretamente identificadas mesmo que não tenham sido adequadamente denominadas.

2. Memória Incidental. Se não tiver identificado alguma figura, explique o que representa. Em seguida, esconda a folha e pergunte: "Que figuras eu lhe mostrei?". O escore é dado pelo número de respostas certas.

3. Memória após 5 minutos. Mostre novamente a folha que contém as figuras durante $\mathbf{3 0}$ segundos dizendo: "Olhe bem e procure guardar na memória." Marque o tempo no relógio e após 5 minutos, pergunte: “Quais as figuras desenhadas que eu lhe mostrei há 5 minutos?”. O escore é dado pelo número de respostas certas.

4. Fluência Verbal. "Você deve falar todos os nomes de animais de que se lembrar no menor tempo possível. Qualquer animal vale: insetos, pássaros, peixes e animais de quatro patas. pode começar." Anote o número de animais nomeados em 1 minuto.

5. Cálculo. Dê uma folha de papel em branco e um lápis ao examinando e peça para fazer os cálculos abaixo. Dite uma conta de cada vez:

$3+2 ; 8+5 ; 32+15 ; 589+234+163$

\section{9-3; 50-14; 549-138}

6. Desenho do relógio. Dê uma folha de papel em branco e um lápis e diga: "Primeiro desenhe um relógio com todos os números. Depois coloque os ponteiros marcando 2 e 45 ". A ordem pode ser repetida, se houver dúvida. Se o desenho for iniciado com um círculo pequeno, peça para fazer um desenho maior.

Escores 10-6: rel6gio e números corretos - 10, hora certa; 9 , leve distúrbio nos ponteiros (por exemplo: ponteiro das horas sobre o 2); 8, distúrbio mais intenso nos ponteiros (por exemplo: anotando 2:20); 7 , ponteiros completamente errados; 6 , uso inapropriado dos ponteiros (por exemplo: uso de código digital ou de círculos envolvendo os números.

Escores 5-1: relógio e números incorretos - 5, números em ordem inversa ou concentrados em alguma parte do relógio; 4, números faltando ou situados fora dos limites do relógio; 3, número e relógio não mais conectados, ausência de ponteiros; 2 , alguma evidência de ter entendido as instruções mas com vaga semelhança com um relógio; 1 , não tentou ou não conseguiu representar um relógio. 
Para comparação de duas ou mais amostras independentes, quando os dados se apresentaram em distribuição ordinal, foi utilizada a prova de Kruskal-Wallis ${ }^{6}$. Para verificar quais os testes neuropsicológicos demonstraram maior acurácia no diagnóstico de demência foram calculadas as áreas sob as curvas ROC (receiver operator characteristics) ${ }^{16,24}$. Para verificar se um conjunto de testes neuropsicológicos permitia distinguir pacientes de controles, foi utilizada análise discriminante ${ }^{17}$. Foi utilizado o programa Epi-Info $5.0^{6}$ para estatística descritiva e prova de Kruskal-Wallis. Para a análise discriminante foi utilizado o programa Statistical Package for the Social Sciences (SPSS) ${ }^{17}$. Admitiu-se nível de significância de 0,05.

\section{RESULTADOS}

Características dos casos e controles estão na Tabela 1. O desempenho nos testes com maior sensibilidade e especificidade diagnósticas estão condensados na Tabela 2. O escore de corte foi definido como aquele que determinava maior valor da soma da sensibilidade e especificidade. Quando mais que 1 escore de corte determinavam somas idênticas, optou-se por aquele em que a especificidade e a sensibilidade tinham valores mais próximos entre si.

A acurácia diagnóstica de cada teste neuropsicológico foi comparada à dos demais através do valor das áreas sob as curvas ROC, que constam da Tabela 3.

A partir da avaliação da sensibilidade, da especificidade e da simplicidade de aplicação, foram escolhidos 6 testes neuropsicológicos que permitem avaliação das seguintes atividades: memória imediata e tardia, atenção, concentração, linguagem, percepção visual, construção, planejamento e cálculo. Os testes selecionados foram os de: fluência verbal, percepção visual, memória incidental, desenho do relógio, cálculo e memória tardia (apos 5 minutos).

Análise discriminante revelou os coeficientes da função discriminante canônica não padronizada e constante que constam da Tabela 4 . Os coeficientes e a constante são aplicados na equação

$\mathrm{D}=$ constante $+0,04866 \mathrm{FV}+(-0,14133) \mathrm{PV}+0,02072 \mathrm{MI}+0,31703 \mathrm{DR}+0,38264 \mathrm{M} 5+(-0,01945) \mathrm{CA}$

onde: D é o escore discriminante; FV, fluência verbal; PV, percepção visual; MI, memória incidental; DR, desenho do relógio; M5, memória após 5 minutos; CA, cálculo.

Aplicando-se essa equação a cada um dos casos e controles, obtêm-se os escores e as relativas probabilidades de classificação correta como caso ou controle, que constam da Figura 1. Todos os indivíduos do grupo controle foram corretamente classificados, obtendo escores positivos. Vinte e nove dos 30 pacientes obtiveram escores negativos; apenas 1 teria sido erroneamente classificado como controle. Quando os testes de cálculo e de percepção visual não foram incluídos, obteve-se outra função discriminante que também classificou corretamente todos os controles e 29 dos 30 doentes.

Escores discriminantes superiores a 1,3 acompanharam-se de probabilidade superior a $99,00 \%$ de classificação correta como controle. Escores negativos e superiores, em números absolutos, a -1,3 acompanharamse de probabilidade superior a 99,00\% de classificação correta como dementes.

Tabela 1. Comparação entre características dos casos e controles quanto a idade, sexo, escolaridade e escore no Mini-exame do Estado Mental.

\begin{tabular}{lccc}
\hline & Casos $(\mathrm{N}=30)$ & Controles $(\mathrm{N}=30)$ & $\mathrm{p}^{*}$ \\
\hline \hline Idade & 52 a 85 anos & 50 a 84 anos & $\mathrm{p}=0,7112$ \\
Média (desvio padrão) & $68,50(8,24)$ & $69,13(7,70)$ & \\
Sexo & 15 masc. $/ 15$ fem. & 15 masc. $/ 15$ fem. & $\mathrm{p}=1,0000$ \\
& 4 a 16 anos & 4 a 16 anos & \\
Escolaridade & $7,30(3,48)$ & $7,36(3,53)$ & $\mathrm{p}=0,8863$ \\
Média (desvio padrão) & 7 a 23 & 25 a 30 & \\
& $16,13(5,16)$ & $28,56(1,38)$ & $\mathrm{p}<0,0000001$ \\
Mini-exame do estado mental & & & \\
Média (desvio padrão) & & & \\
\hline
\end{tabular}

*prova de Kruskal-Wallis. 
Tabela 2. Escores máximos e mínimos, médias e desvios padrões, escores de corte, sensibilidade e especificidade de alguns testes neuropsicológicos aplicados nos casos e nos controles.

\begin{tabular}{|c|c|c|c|c|c|c|c|}
\hline \multirow{2}{*}{ Teste } & \multicolumn{2}{|c|}{ Casos } & \multicolumn{2}{|c|}{ Controles } & \multirow{2}{*}{ Corte } & \multirow{2}{*}{$\begin{array}{l}\text { Sens. } \\
(\%)\end{array}$} & \multirow{2}{*}{$\begin{array}{c}\text { Espec } \\
(\%)\end{array}$} \\
\hline & $\begin{array}{l}\text { Min / } \\
\text { Máx. }\end{array}$ & $\begin{array}{l}\text { Média } \\
\text { (DP) }\end{array}$ & $\begin{array}{l}\text { Min / } \\
\text { Máx. }\end{array}$ & $\begin{array}{l}\text { Média } \\
\text { (DP) }\end{array}$ & & & \\
\hline $\begin{array}{l}\text { Blessed } \\
\text { (cognitivo) }\end{array}$ & $8-32$ & $\begin{array}{l}19,23 \\
(6,31)\end{array}$ & $29-37$ & $\begin{array}{l}34,23 \\
(2,04)\end{array}$ & $<29$ & 96,66 & 100 \\
\hline $\begin{array}{l}\text { Dígitos } \\
\text { (ordem direta) }\end{array}$ & $2-7$ & $\begin{array}{c}4,06 \\
(1,20)\end{array}$ & $4-7$ & $\begin{array}{c}5,20 \\
(0,80)\end{array}$ & $<5$ & 60 & 80 \\
\hline $\begin{array}{l}\text { Dígitos } \\
\text { (ordem inversa) }\end{array}$ & $0-4$ & $\begin{array}{c}1,80 \\
(1,40)\end{array}$ & $2-5$ & $\begin{array}{c}3,60 \\
(0,77)\end{array}$ & $<3$ & 60 & 96,66 \\
\hline $\begin{array}{l}\text { Conexão de } \\
\text { números }\end{array}$ & $85-305$ & $\begin{array}{l}248,96 \\
(78,76)\end{array}$ & $30-170$ & $\begin{array}{c}75,70 \\
(34,39)\end{array}$ & $>120$ & 90 & 86,66 \\
\hline Fluência verbal & $2-13$ & $\begin{array}{c}6,60 \\
(3,40)\end{array}$ & $5-29$ & $\begin{array}{l}17,93 \\
(5,08)\end{array}$ & $<13$ & 96,66 & 93,33 \\
\hline Percepção visual & $2-10$ & $\begin{array}{c}7,46 \\
(2,93)\end{array}$ & $8-10$ & $\begin{array}{c}9,73 \\
(0,52)\end{array}$ & $<9$ & 50 & 96,66 \\
\hline Memória incidental & $0-6$ & $\begin{array}{c}2,23 \\
(1,61)\end{array}$ & $3-10$ & $\begin{array}{c}5,70 \\
(1,39)\end{array}$ & $<5$ & 90 & 83,33 \\
\hline $\begin{array}{l}\text { Memória verbal } \\
\text { após } 5 \text { minutos }\end{array}$ & $0-7$ & $\begin{array}{c}1,86 \\
(2,11)\end{array}$ & $5-10$ & $\begin{array}{c}7,66 \\
(1,29)\end{array}$ & $<6$ & 93,33 & 96,66 \\
\hline $\begin{array}{l}\text { Memória verbal } \\
\text { após } 15 \text { minutos }\end{array}$ & $0-9$ & $\begin{array}{c}1,26 \\
(2,06)\end{array}$ & $5-10$ & $\begin{array}{c}7,56 \\
(1,33)\end{array}$ & $<5$ & 93,33 & 100 \\
\hline Reconhecimento & $0-9$ & $\begin{array}{c}4,90 \\
(2,99)\end{array}$ & $8-10$ & $\begin{array}{c}9,63 \\
(0,66)\end{array}$ & $<9$ & 93,33 & 90 \\
\hline Construção & $1-11$ & $\begin{array}{c}6,26 \\
(3,09)\end{array}$ & $0-1$ & $\begin{array}{c}0,25 \\
(0,50)\end{array}$ & $>1$ & 96,66 & 100 \\
\hline Desenho do relógio & $0-8$ & $\begin{array}{c}3,30 \\
(1,98)\end{array}$ & $5-10$ & $\begin{array}{c}8,23 \\
(1,54)\end{array}$ & $<5$ & 90 & 83,33 \\
\hline
\end{tabular}


Tabela 3. Valores das áreas sob curva ROC de alguns testes neuropsicológicos.

\begin{tabular}{lc}
\hline \multicolumn{1}{c}{ Teste } & Área \\
\hline \hline Blessed cognitivo & 0,9942 \\
Construção & 0,9914 \\
Memoria verbal (5 minutos) & 0,9834 \\
Reconhecimento & 0,9809 \\
Fluência verbal & 0,9764 \\
Conexão de números & 0,9721 \\
Memória verbal (15 minutos) & 0,9621 \\
Desenho do relógio & 0,9614 \\
Memória verbal (30 minutos) & 0,9597 \\
Memória verbal incidental & 0,9458 \\
Cálculo & 0,9215 \\
\hline
\end{tabular}

Tabela 4. Valores dos coeficientes da Função Discriminante Canônica Não Padronizada (e da constante).

\begin{tabular}{lc}
\hline Fluência verbal (FV) & 0,04866 \\
Percepção visual (PV) & $-0,14133$ \\
Memória verbal incidental (MVI) & 0,02072 \\
Desenho do relógio (DR) & 0,31703 \\
Memória verbal após 5 minutos & \\
(MV5) & 0,38264 \\
Cálculo (CA) & $-0,01945$ \\
Constante & $-3,01632$ \\
\hline
\end{tabular}

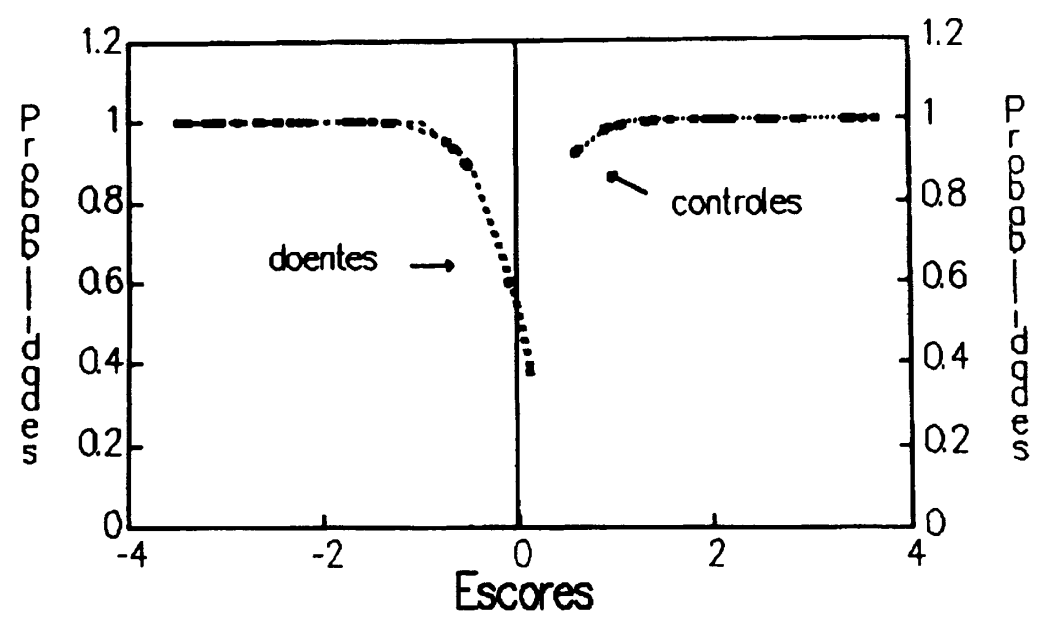

Fig 1. Escores da função linear discriminante e probabilidades de classificação correta como doente ou controle.

\section{COMENTÁRIOS}

Para verificar quais os testes com maior acurácia diagnostica foram comparadas as áreas sob as curvas ROC. Este método tem sido utilizado em estudos semelhantes e é superior à simples avaliação das sensibilidade e especificidade obtidas com um único valor de corte ${ }^{14,16,24}$. Quanto maior a área sob a curva, maior a acurácia do teste.

Dentre os testes empregados, o que demonstrou maior acurácia foi o teste informação-memóriaconcentração de Blessed (Blessed cognitivo). Quando o escore de corte foi de 28/29, apenas 1 paciente teria sido incorretamente classificado como normal. Este teste é semelhante ao MEM, com as diferenças de que não avalia habilidades construtivas, cálculo e alguns itens da linguagem, como nomeação de objetos e repetição de frase. Ambos apresentam correlação elevada ${ }^{8}$.

Em seguida, o teste com maior acurácia foi o de construção, em que o indivíduo deve copiar 6 desenhos, 4 destes propostos por Rosen et al. ${ }^{20}$, e desenhar espontaneamente uma casa. Os pacientes 
e alguns indivíduos do grupo controle apresentaram muita dificuldade ao copiar a figura de um cubo. Mas 29 dentre 30 pacientes cometeram mais do que 1 erro nesses desenhos enquanto nenhum indivíduo do grupo controle errou mais do que 1.

A seguir, os testes que revelaram maior acurácia foram os de memória verbal tardia (após 5 minutos) seguido pelo de reconhecimento das 10 figuras que haviam sido apresentadas $\mathbf{3 0}$ minutos antes. Estes dados são semelhantes aos de Welsh et al. ${ }^{29}$, para quem a memória verbal tardia foi o melhor teste para discriminar pacientes com DA de indivíduos controle. Em nossa avaliação, as 10 palavras são apresentadas como figuras, para permitir o exame de indivíduos analfabetos e para avaliar a percepção visual.

O teste de fluência verbal, número de animais nomeados em 1 minuto, também revelou acurácia elevada. Apenas 1 paciente conseguiu dizer o nome de mais de 12 animais em 1 minuto; por outro lado, somente 2 indivíduos do grupo controle disseram menos de 13 nomes. Quando comparado a outros testes de fluência verbal, os que avaliam categoria (animais, itens de vestuário, vegetais, frutas) têm se demostrado superiores aos demais (por exemplo: palavras iniciadas com determinada letra) $)^{14}$.

Em ordem decrescente de acurácia, situaram-se os testes de conexão de números, memória verbal após 15 minutos, desenho do relógio, memória verbal com 30 minutos, memória incidental, cálculo e os testes de memória espacial após 5 e após 15 minutos. Testes de retenção de dígitos, em ordem direta ou inversa, revelaram pequena capacidade discriminativa.

Os testes escolhidos para a análise discriminante foram os de fluência verbal (animais), percepção visual, memória visual incidental, cálculo, desenho do relógio e memória verbal tardia após 5 minutos. Estes testes foram escolhidos porque podem ser aplicados em pessoas analfabetas ou de baixa escolaridade e porque alguns destes testes são complementares quanto à técnica de aplicação. Por exemplo, a percepção visual das figuras e a memória incidental necessariamente precedem o teste de memória tardia das mesmas figuras. Entre a percepção visual e o teste de memória tardia há intervalo de 5 minutos a preencher. Os testes de cálculo e do desenho do relógio, que são de interpretação mais simples que os de construção, podem ser aplicados nesse intervalo.

A função linear discriminante obtida fornece um escore que permite avaliar a probabilidade de ser um indivíduo corretamente classificado como caso ou controle. Como exemplo, considere-se um dos casos cujos escores foram: fluência verbal $(F V)=7$; percepção visual $(P V)=9$; memória incidental $(M I)=2$; desenho do relógio $(D R)=5$; memoria tardia $(M 5)=0$ e cálculo $(C A)=7$. Aplicandose a função discriminante (com aproximação até a segunda casa decimal), com os valores da constante e dos coeficientes indicados na Tabela 4, obtém-se o escore discriminante (D):

$\mathrm{D}=-3,02+0,05 \mathrm{FV}+(-0,14) \mathrm{PV}+0.02 \mathrm{MI}+0,32 \mathrm{DR}+0,38 \mathrm{M} 5+(-0,02) \mathrm{CA}$

$\mathrm{D}=-3,02+(0,05 \times 7)-(0,14 \times 9)+(0,02 \times 2)+(0,32 \times 5)+(0,38 \times 0)-(0,02 \times 7)$

$D=-2,43$

que corresponde a probabilidade superior a $99,00 \%$ de classificação correta como demente.

Esta função deve ser submetida à comprovação em outra casuística. Se comprovada, poderá ser utilizada para exame de um paciente. Mas seu valor deverá ser maior para avaliar amostras populacionais. Em virtude da simplicidade e rapidez de aplicação, poderá ser associado ao MEM para identificar indivíduos com demência e indivíduos sadios em uma amostra populacional. Há necessidade de aplicá-la a grupos com escolaridade mais baixa para verificar se mantém capacidade discriminativa. 
Outra possibilidade de pesquisa, de interesse prático, consiste em verificar se pacientes com demência muito leve ou questionável podem ser distinguidos de normais, com este exame, em estudos de coorte. Estudos recentes têm verificado que as mesmas funções psicológicas estão comprometidas nas síndromes demenciais leves e muito leves ${ }^{16,22}$, mas que existe muita superposição entre escores obtidos pelos indivíduos normais e pelos pacientes com demência muito leve.

\section{REFERÊNCIAS}

1. American Psychiatric Association. Diagnostic and statistical manual of mental disorders. Ed 3 rev. Washington, DC: American Psychiatric Association, 1987.

2. Anthony JC, LeResche L, Niaz U, Von Körff MR, Folstein M. Limits of the "Mini-mental state" as a screening test for dementia and delirium among hospital patients. Psychol Med 1982, 12: 397-408.

3. Blessed G, Tomlinson BE, Roth $M$. The association between quantitative measures of dementia and of senile change in the cerebral grey matter of elderly subjects. Br J Psychiatry 1968, 114: 797-811.

4. Conn HO. Trailmaking and number-connection tests in the assessment of mental state in portal systemic encephalopathy. Dig Dis 1977, 22: 541-550.

5. Crystal HA, Dickson DW, Lizardi JE, Davies P, Wolfson LL. Antemorten diagnosis of diffuse Lewy body disease. Neurology 1990, 40: 1523-1528.

6. Dean AG, Dean JA, Burton AH, Dicker RC. Epi Info, version 5: a word processing, database, and statistics program for epidemiology on micro-computers. Atlanta: Centers for Disease Control, 1990.

7. Eslinger PJ, Damasio AR, Benton AL, Van Allen M. Neuropsychologic detection of abnormal mental decline in older persons. JAMA 1985, 253: 670-674.

8. Fillenbaun GG, Heyman A, Wilkinson WE, Haynes CS. Comparison and reliability of the mini-mental state examination and the modified Blessed test. Arch Neurol 1987, 44: 924-927.

9. Folstein MF, Folstein SE, McHugh PR. "Mini-mental state": a practical method for grading the coignitive state of patients for the clinician. J Psychiatr Res 1975, 12: 189-198.

10. Galasko D, Klauber MR, Hofstetter R, Salmon DP, Lasker B, Thal LJ. The mini-mental state examination in the early diagnosis of Alzheimer's disease. Arch Neurol 1990, 47: 49-52.

11. Lefevre BH, Nitrini R. Semiologia neuropsicológica. Arq Neuropsiquiatr 1985, 43: 119-132.

12. McKeith LG, Perry RH, Fairbairn AF, Jabeen S, Perry EK. Operacional criteria for senile dementia of Lewy body type (SDLT). Psychol Med 1992, 22: 911-922.

13. McKhann G, Drachman D, Folstein M, Katzman R, Price D, Stadlan EM. Clinical diagnosis of Alzheimer's disease. Neurology 1984, 34: 939-944

14. Monsch AU, Bondi MW, Butters N, Salmon DP, Katzman R, Thal LJ. Comparisons of verbal fluency tasks in the detection of dementia of the Alzheimer type. Arch Neurol 1992, 49: 1253-1258.

15. Morris JC, McKeel DW, Storandt M, Rubin EH, Price JL, Grant EA, Ball MJ, Berg L. Very mild Alzheimer's disease: informant-based clinical, psychometric, and pathologic distinction from normal aging. Neurology 1991, 41: 469-478.

16. Mossman D, Somoza E. ROC curves, test accuracy, and the description of diagnostic tests. J Neuropsychiatry 1991, 3: 330-333.

17. Nie NH, Hull CH, Jenkins JG, Steinbrenner K, Bent D. Statistical package or the social sciences. Ed 2, New York: McGraw-Hill, 1975.

18. Nitrini R. Diagnóstico de demência; avaliação clínica, neuropsicológica e através da tomografia computadorizada por emissão de fóton único. Tese de Livre-Docência. Faculdade de Medicina da Universidade de São Paulo, São Paulo, 1993.

19. Román GC, Tatemichi TK, Erkinjuntti T, Cummings JL, Masdeu JC, Garcia JH, Amaducci L, Orgogozo JM, Brun A, Hofman A, Moody DM, O'Brien MD, Yamaguchi T, Grafman J, Drayer BP, Bennett DA, Fischer M, Ogata J, Kokmen E, Bermejo F, Wolf PA, Gorelick PB, Bick KL, Pajeau AK, Bell MA, DeCarli C, Culebras A, Korczyn ADS, Bougousslavsky J, Hartmann A, Cheinberg P. Vascular dementia: diagnostic criteria for research studies. Neurology 1993, 43: 250-260.

20. Rosen WG, Mohs RC, Davis KL. A new rating scale for Alzheimer's disease. Am J Psychiatry 1984, 141: 1356-1364.

21. Storandt M, Botwinick J, Danziger WL, Berg L, Hughes CP. Psychometric differentiation of mild senile dementia of the Alzheimer type. Arch Neurol 1984, 41: 497-499.

22. Storandt M, Hill RD. Very mild senile dementia of the Alzheimer type: psychometric test performance. Arch Neurol 1989, 46: 383-386.

23. Sunderland T, Hill JL, Mellow AM, Lawlor BA, Gundersheimer J, Newhouse PA, Grafman JH. Clock drawing in Alzheimer's disease: a novel measure of dementia severity. J Am Geriatr Soc 1989, 37: 725-729. 
24. Swets JA. Measuring the accuracy of diagnostic systems. Science 1988, 240: 1285-1293.

25. Tierney MC, Snow G, Reid DW, Zorzitto ML, Fisher RH. Psychometric differentiation of dementia: replication and extension of the findings of Storandt and coworkers. Arch Neurol 1987; 44: 720-722.

26. Uhlmann RF, Larson EB. Effect of education on the mini-mental state examination as a screening test for dementia. J Am Geriatr Soc 1991, 39: 876- 880.

27. Wechsler D. A standardized memory scale for clinical use. J Psychology 1945, 19: 87-95.

28. Weintraub S, Mesulam MM. Mental state assessment of young and elderly adults in behavioral neurology. In Mesulam MM. Principles of behavioral neurology. Philadelphia: Davis, 1985: 71-123.

29. Welsh K, Butters N, Hughes J, Mohs R, Heyman A. Detection of abnormal memory decline in mild cases of Alzheimer's disease using CERAD neuropsychological measures. Arch Neurol 1991, 48: 278-281. 